\title{
State of the Art and Research Challenges for USN Traffic Flow Models
}

\author{
Andrey Koucheryavy* \\ *State University of Telecommunication, Pr. Bolshevikov, 22, St.Petersburg, Russia \\ Central Science Research Telecommunication Institute, First Proezd Perova Polya, 8, Moscow. Russia \\ akouch@mail.ru
}

\begin{abstract}
The traffic flow model was one of the most important investigation topic for any networks during them evolution. The traffic flow model understanding was changes from network to network, from service to service. The USN traffic flow models are in the focus of this paper. The telemetry, image and medical USN applications traffic flow models are investigated. The selfsimilarity features are determined. The practical benefits of obtained results are considered. The traffic flow type impact to the USN life-time in the different intrusion conditions is proved.
\end{abstract}

\section{Keywords - USN, traffic flow, Hurst parameter}

\section{INTRODUCTION}

The 7 trillion wireless telecommunication units for 7 billion people could be form the network at 2017-2020 in according with forecast [1]. Moreover, the estimation of the total number of things that could be connected with the future networks is 50 trillion (up to 5000 things for every human) [2]. Therefore the future networks will be self-organizing and the most important traffic sources will be things.

The Internet of Things (IoT) is the new ITU-T concept for the network develop-ment. The ITU-T determines IoT as a future global infrastructure: "In a broad per-spective, the IoT can be perceived as a vision with technological and societal implica-tions. From the perspective of technical standardization IoT can be viewed as a global infrastructure for the information society, enabling advanced services by interconnecting (physical and virtual) things based on, existing and evolving, interoperable information and communication technologies" $[3,4]$. Things are considered as things from the nature and information worlds.

The IoT are based on the Ubiquitous Sensor Network (USN). So the USN traffic models should be studied well. The traffic flow model was one of the most important investigation topic for any networks during them evolution. The traffic flow model understanding was changes from network to network, from service to service.

The voice traffic arrival process for circuit switched public telecommunication net-works is the Poisson process based on long-term observation.

The fundamental properties of the Poisson process are stationary, independent at all time instants (epochs), and simple. The following two most important Poisson process properties could be considered [5]:
- the number of events within a time interval of fixed length is Poisson distributed,

- the time distance between consecutive events is exponentially distributed.

The Poisson flow is fully determined by its own parameter.

The Poisson arrival process is adequate for submission of other arrival processes, for example the dial-up Internet access arrival process [5].

Both the data and video traffic creating the new types of arrival traffic flows in NGNs are self-similar traffic flows.

The arrival traffic process is considered as self-similar if the distribution function for the original and the aggregated processes are equal. The aggregated arrival process is created by the averaging of the original process in $\mathrm{m}$ size series blocks (the block is part of the original process) [6].

The most important feature of the self-similar arrival process is the Hurst parameter [7]. Knowledge of the Hurst parameter value gives the possibility to generate the selfsimilar flow with specified characteristics.

The following traffic flow characteristics were obtained. The "www" traffic is self-similar with the Hurst parameter $\mathrm{H}$ $=0.7-0.9$ [8] and [9]. File transfer traffic is self-similar too with the Hurst parameter $\mathrm{H}=0.85-0.95$ [10] and [11]. E-mail traffic is self-similar with the Hurst parameter $\mathrm{H}=0.75$ [9]. As we see the general data traffic types in the IP networks are the self-similar with high values of Hurst parameter.

But the statistical observation of VoIP traffic flows on the real networks proved that the arriving VoIP traffic flow could be described by the Poisson process. Furthermore, the Poisson traffic flow model could be used for VoIP traffic, even for allIP networks [12].

The IPTV traffic flow statistical observation proved that the traffic flow is self-similar both for multicast and unicast traffic [13], [14] and [15]. The Hurst parameter values are $\mathrm{H}=$ $0.55-0.6$ for multicast traffic and $\mathrm{H}=0.75-0.8$ for unicast traffic.

Furthermore, peer-to-peer traffic is self-similar. The Skype traffic as P2P traffic example is self-similar with $\mathrm{H}=0.6$ for inter-arrival time and $\mathrm{H}=0.7$ for packet lengths [16].

We will focus on the USN traffic flow models further. The Poisson arrival process was assumed as traffic model for each individual sensor node in [17]. The ON/OFF method [6] for USN traffic models was analyzed in [18]. Authors proved that 
ON period distribution and OFF period distribution could be described by generalized Pareto distribution. The pseudo long range dependent (LRD) traffic model was pro-posed in [19] for mobile sensor networks.

There are many USN classifications. The appendix 1 to [20] determines the USN classification by such objects characteristics as size, mobility, power, connectivity, ability, people involvement, physical/logical objects, object with tag, IP/non-IP. The USN traffic flow models for telemetry, images and medical applications will study further. The traffic flow type impact to the USN life-time in the different intrusion conditions will study too.

\section{TELEMETRY APPLICATIONS}

The USN traffic flow models for Telemetry applications are studying further both for fixed wireless sensor nodes and mixed fixed/mobile wireless sensor nodes [21].

The network model for fixed wireless sensor nodes includes 50 nodes, which randomly located on the plane size $30 * 30 \mathrm{~m}$. The sink is on plane centre. This model is shown in the fig.1. The above mentioned parameters are typically for warehouse. Each sensor node sends the messages every 15, 30, 45 or $60 \mathrm{~s}$ in according with given initial random distribution.

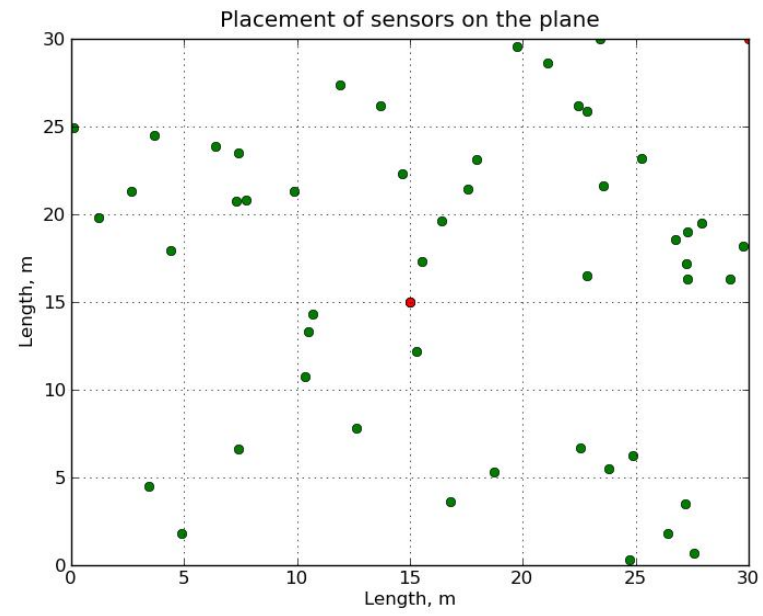

Figure 1. Placement of sensors on the plane.

The modelling was held by ns-2.The data processing was made by Python language libraries Numpy and Scipy. The data visual process was support by library Matpplotlib. The modelling main goal is the USN traffic self-similar detection and Hurst parameter determination.

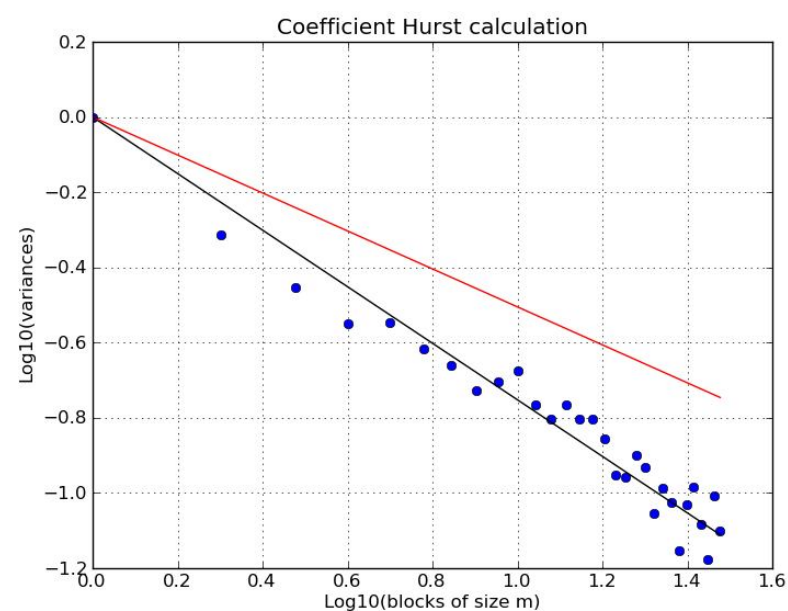

Figure 2. Hurst coefficient calculation based on the line slope

The Hurst parameter mean value estimation is $\mathrm{H}=0.675$. This value was calculated by 1000 time realization of random process. So, the USN traffic for telemetry applications based on fixed sensor nodes is the self-similar with middle level self-similarity.

The network model for the mixed network is the similar as for fixed nodes, but for half of nodes request the movement each $50 \mathrm{~s}$. The movement rate is $2 \mathrm{~m} / \mathrm{s}$ [22].

The Hurst parameter mean value estimation is $\mathrm{H}=0.687$. The Hurst parameter mean value estimation is $\mathrm{H}=0.675$. This value was calculated by 3000 time realization of random process. So, the USN traffic for telemetry applications based on mixed fixed/mobile sensor nodes is the self-similar with middle level self-similarity.

The Hurst parameter measured values are shown in the fig.3. There are two clusters in the fig.3. One of them content the reconfiguration and signaling traffic only (up to 2200 events), another cluster includes all types of traffic reconfiguration, signaling and information (telemetry data).

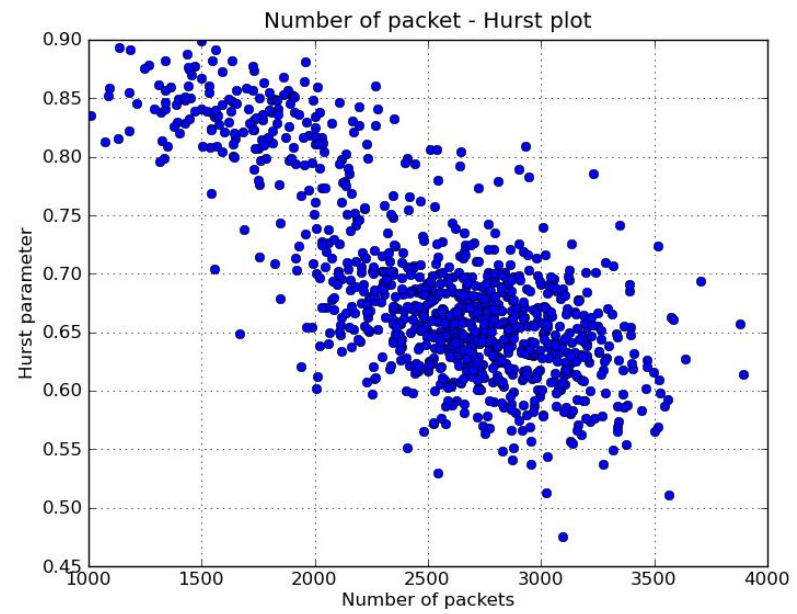

Figure 3. Placement of sensors on the plane. 
The Hurst parameter mean value estimation for reconfiguration and signaling traffic is $\mathrm{H}=0.829$. So, the USN traffic for reconfiguration and signaling in the telemetry applications based on mixed fixed/mobile sensor nodes is the self-similar with high level self-similarity.

\section{THE IMAGE APPLICATIONS}

The sensor field of 30 by 30 meters was selected as base model for investigated USN likely previous experience. Two types nodes randomly located in the network. The first type of sensors is nodes that gathering telemetry data, whose task is to transfer small amounts of data with a given period. The transmission frequency is determined for each node type accidentally ranging from 15 to 60 seconds in steps of 15 seconds. The second type of sensor node is transmitting images. Each of these nodes transmits at a random time interval batch of packets that simulate the transfer of photos size of $640 \times 480$ pixels. The amount of data transmitted is randomly selected based on the evaluation of the results get in [23].

The Hurst parameter values as a function from number of sensors which trans-mitting images [24]. The overload condition is a state when the network can't handle fully photo transfer. It is easily seen that the Hurst coefficient reaches its maximum in about 30 nodes - transmitters (fig.4). It's clear that the result of changes in the structure of traffic, and therefore the Hurst coefficient changes is caused by network overloading.

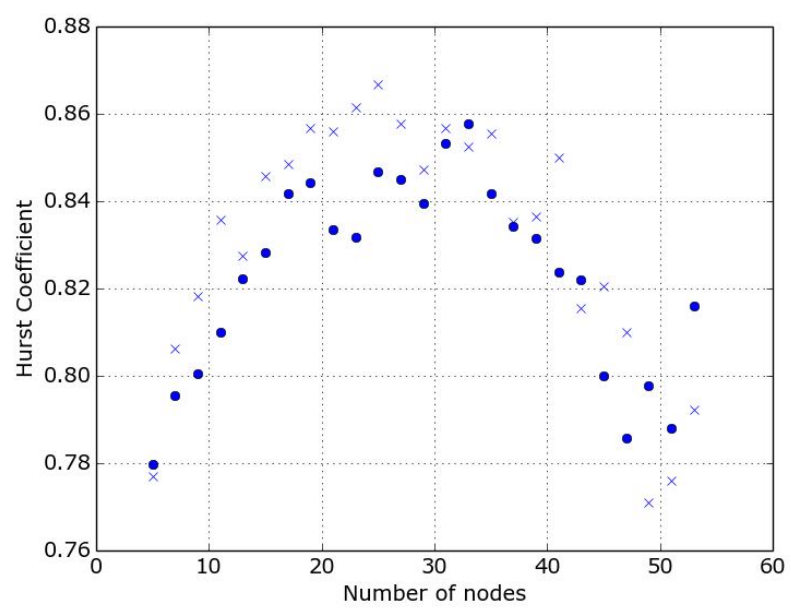

Figure 4. The Hurst coefficient as a function from the number of photo transmitters

In any case, the traffic flow for USN image applications is the self-similarity with high value of Hurst parameter.

\section{IV.MEDICAL APPLICATIONS}

The medical USN intended for communication between hospital data centre and medical devices for continuous health monitoring (e.g. pulsometers, sphygmomanometers, and modern glucometers) is studied in [25]. The network architecture in this case is hybrid: mobile health sensors periodically send data to the traffic sink through the network composed of stationary nodes. The application scenario is periodic; therefore source traffic could be modelled by Constant Bit Rate (CBR) [18].

The self-similarity of USN traffic in this application described above was taken as a hypothesis. To test this assumption we used two methods for self-similarity level estimation: the R/S and the Higuchi methods.

For the medical USN simulation showed the Hurst parameter dependence on the interval between packet sending. For the 20 bytes packets and the intervals between packets less than 5 seconds increase of the Hurst parameter were shown. For the intervals grater then 5 seconds the Hurst parameter doesn't change. The R/S method estimation of the Hurst parameter is 0.66 in stationary state; the Higuchi method estimation is 0.76 .

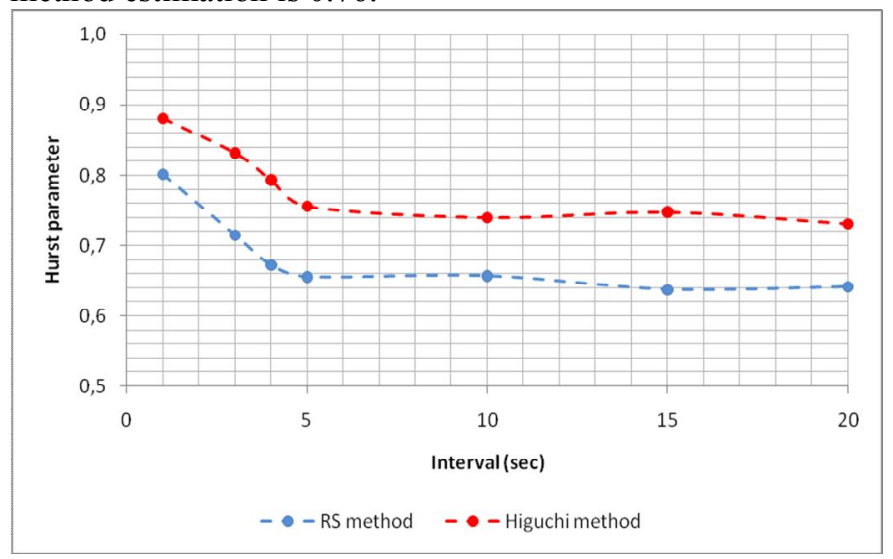

Figure 5. Medical USN. Hurst parameter dependence on the length of interval between packets.

As we see the Hurst parameter values for the medical applications can be change from small up to high.

\section{THE TRAFFIC FLOW TYPE IMPACT TO THE USN LIFE- TIME IN THE DIFFERENT INTRUSION CONDITIONS}

Let's consider the practical benefits of obtained results. The traffic flow type impact to the USN life-time in the different intrusion conditions will investigate. The network-based security for sensor networks has specific features. These features are covered in ITU-T Recommendation X.1311 [26]. First of all, we should note that an enormous number of sensor nodes and hard energy constraints are the most important features of sensor networks. The more than 64000 nodes could be organized on a single sensor network in accordance with ZigBee protocol specifications [27]. The energy consumption is the most important parameter for sensor network life-time and for modern networks in whole [28, 29,30]. The connectivity, mobility and coverage can also considered as important parameters [22].

An enormous number of sensor nodes and high requirements to energy system de-fine specific features for sensor network-based security. The specific network-based security support topics for the sensor networks include in accordance with Recommendation X.1311:

- difficulty of using public key cryptosystems,

- vulnerability of sensor nodes, 
- difficulty in obtaining post deployment knowledge,

- limited memory size, transmission power, and transmission bandwidth,

- single point of failure of a base station.

The last item relates to a cluster head node too.

Sensor nodes are very simple devices. Cloning is the one more specific threat for sensor networks [31]. The sensor network energy system is very vulnerable. The energy system conditions are directly connected to the sensor network lifetime. For example, attacks to the sensor network energy system can be organized as the sensor nodes sleep deprivation [32]. One more type of attacks to the sensor network energy system, which is based on the generation of spurious flows, was proposed in the [33]. The sensor nodes respond to the spurious flows and the sensor network life-time de-creases. The mobile sensor network behavior in the case of the spurious flows intrusion was investigated in the [33].

Let's consider the next model. The 100 sensor nodes are distributed on the plane 200x200m randomly. The sensor node activity radius is $25 \mathrm{~m}$. The sensor nodes average moving speed varies from $1 \mathrm{~m} / \mathrm{s}$ (slowly walking pedestrian) up to 8 $\mathrm{m} / \mathrm{s}$ (average car speed in the big town). The initial energy is $2 \mathrm{~J}$ for each sensor node, the energy consumption for receiving is $50 \mathrm{~nJ} / \mathrm{bit}$, the energy consumption for transmitting is 50 $\mathrm{nJ} / \mathrm{bit}$ and $100 \mathrm{pJ}$ per square meter additionally. The sink located in the plane centre. The cluster head selection algorithm is LEACH.

The spurious flow two types are considered. One of them is the Poisson flow, an-other flow type is deterministic. The spurious flows intensity varies from 1 event/s up to 10 events/s. The C\#.NET is used for modelling.

The life-time in rounds as a function of the spurious flow intensity for different moving speeds of sensor nodes in case of deterministic $(2 \mathrm{~m} / \mathrm{s})$ and Poisson $(2,4,6$, and $8 \mathrm{~m} / \mathrm{s})$ flows is shown in Fig. 6. First of all we note that spurious flows intrusion reduce the sensor network life-time. Furthermore, the type of spurious flows also affects the sensor network lifetime. This is especially noticeable in the range from 1 to 2 event/s. In this range, deterministic flows cause a more dramatic decrease in the sensor network life-time than Poisson ones. Thus, it is possible to adjust the spurious flow intensity and the type of flows to maximize the impact of the attack on the sensor network life-time.

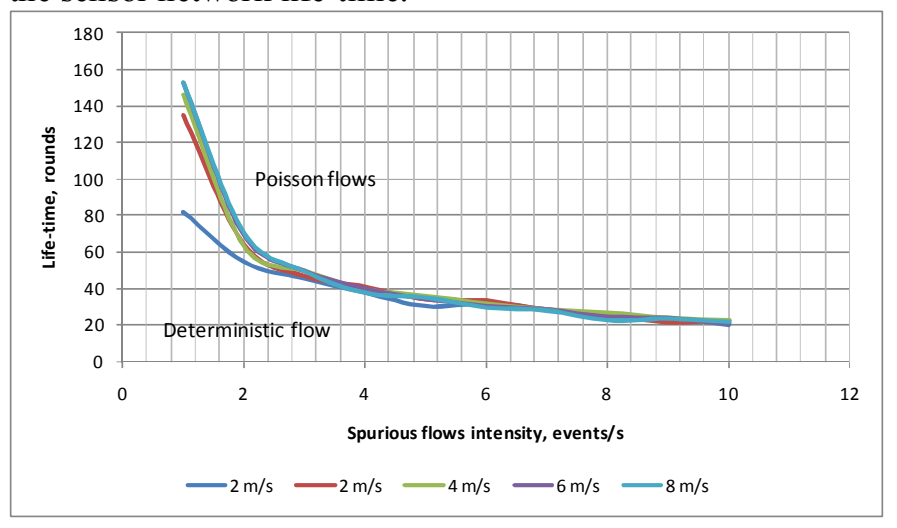

Figure 6. The life-time in rounds as a function of the spurious flow intensity for different moving speeds of sensor nodes in case of deterministic $(2 \mathrm{~m} / \mathrm{s})$ and Poisson $(2,4,6$, and $8 \mathrm{~m} / \mathrm{s})$ flows.

\section{Conclusions}

The USN traffic for reconfiguration and signalling in the telemetry applications based on mixed fixed/mobile sensor nodes is the self-similar with high level self-similarity. The traffic flow for USN image applications is the self-similar with high value of Hurst parameter. The Hurst parameter values for the medical applications can be change from small up to high.

The deterministic flows cause is a more dramatic decrease in the sensor network life-time than Poisson ones. Thus, it is possible to adjust the spurious flow intensity and the type of flows to maximize the impact of the attack on the sensor network life-time.

\section{REFERENCES}

[1] L.Sorensen, K.E.Skouby. "Use scenarios 2020 - a worldwide wireless future. Visions and research directions for the Wireless World." Outlook. Wireless World Research Forum. July 2009, №4.

[2] J.-B.Waldner. "Nanocomputers and Swarm Intelligence." ISTE, Wiley\&Sons, London, 2008, p.212.

[3] Recommendation Y.2060. Overview of Internet of Things. ITU-T, February 2012, Geneva.

[4] A.Iera, C.Floerkemeier, J.Mitsugi, G.Morabito. "The Internet of Things." IEEE Wireless Communications. Dec. 2010, v.17,\#6.

[5] V.B.Iversen. Teletraffic Engineering and Network Planning, Technical University of Denmark, $<\underline{\text { http://www.osti.gov/eprints/topicpages/documents/record/982/147313 }}$ 2.html >, 2010.

[6] W.Willinger, M.Taqqu, R.Sherman, D.Wilson. "Self-similarity through High-variability" in IEEE/ACM Transaction on Networking.,v.15, \#1,1997.

[7] O.Sheluhin, S Smolskiy, A.Osin, A. Self-Similar Processes in Telecommunications. Wiley, 2007.

[8] M.E.Crovella, A.Bestavros. Self-Similarity in Wide Web Traffic: Evidence and Possible Causes. IEEE/ACM Transaction on Networking, v.5, No. 6, December 1997.

[9] J.Ho, Y.Zhu, S.Madhavapaddy. Throughput and buffer analysis for GSM general packet radio service, Proceedings WCNC'99, New Orleans, USA, September 1999.

[10] I.Norros. The Management of Large Flows of Connectionless Traffic on the Basis of Self-similar Modeling, International Conference on Communications ICC'95. Proceedings, 18-22 June 1995, Seattle, USA.

[11] A.K.Jena, P.Pruthi,A. Popesku. Modelling and Evaluation of Network Applications and Services, Blekinge Institute of Technology, Karlskrona, Sweden. < www.bth.se $>, 2011$.

[12] R.Birke, M.Mellia, M.Petracca, D.Rossi. Experience of VoIP Traffic Monitoring in a Commercial ISP, International Journal of Network Management, vol. 20, Issue 5, 2010.

[13] T.Janevski, Z.Vanevski. Statistical Analysis of Multicast versus Instant Channel Changing Unicast IPTV Provisioning, 16th Telecommunications Forum TELFOR 2008, Belgrade, Serbia, 25-27 November 2008.

[14] D.Tarasov, A.Paramonov, A.Koucheryavy. The Video Streaming Monitoring in the Next Generation Network. LNCS, Springer, volume 5764 Smart Spaces and Next Generation Wired/Wireless Networking, 2010 .

[15] M.Mellia, M.Meo. Measurement of IPTV Traffic from an Operative Network, European Transactions on Telecommunications, 2009.

[16] N.M.Markovich, U.R.Krieger. Statistical Analysis and Modeling of Peer-to-Peer Multimedia Traffic, LNCS 5233, Next Generation Internet (Ed. D.Kouvatsos), 2011.

[17] S.Tang. An Analytical Traffic Flow Model for Cluster-Based Wireless Sensor Networks. 1st International Simposium on Wireless Pervasive Computing, 2006. 
[18] Q.Wang, T.Zhang. "Source Traffic Modelling in Wireless Sensor Networks for Target Tracking" in 5th ACM International Simposium on Performance Evaluation of Wireless Ad Hoc, Sensor and Ubiquitous Networks (PEWASUN'08), 27-31 October, 2008, Vancouver, Canada Proceedings.

[19] P.Wang, I.F.Akyildiz. Spatial Correlation and Mobility Aware Traffic Modelling for Wireless Sensor Networks. IEE Global Communications Conference (GLOBECOM"09), 30 November - 4 December, 2009, Honolulu, Havaii, USA, Proceedings.

[20] Recommendation Y.2062. Framework of object-to-object communication using ubiquitous networking in NGN. ITU-T, Feb. 2012. Geneva.

[21] A.Koucheryavy, A.Prokopiev. "Ubiquitous Sensor Networks Traffic Models for Telemetry Applications" in The 11th International Conference on Next Generation Wired/Wireless Networking NEW2AN 2011. Aug. 2011 Saint-Petersburg. Springer LNCS 6869.

[22] A. Koucheryavy, A. Salim. Prediction-based Clustering Algorithm for Mobile Wireless Sensor Networks. Proceedings, International Conference on Advanced Communication Technology, 2010. ICACT 2010. Phoenix Park, Korea.

[23] A.Koucheryavy, A.Muthanna, A.Prokopiev. Ubiquitous Sensor Networks Traffic Models for Image Applications. Internet of Things and its Enablers (INTHITEN). Conference, State University of Telecommunication, St. Petersburg, Russia, June 3-4, 2013. Proceedings.

[24] A.Koucheryavy, A.Muthanna, A.Prokopiev. The Mixed Telemetry/Image USN in the Overload Conditions. Proceedings, International Conference on Advanced Communication Technology, 2014. ICACT 2014, Phoenix Park, Korea.
[25] A.Koucheryavy, A.Vybornova. "Ubiquitous Sensor Networks Traffic Models for Medical and Tracking Applications" in The 12th International Conference on Next Generation Wired/Wireless Networking NEW2AN 2012. Aug. 2012 Saint-Petersburg. Springer LNCS 7469.

[26] Recommendation X.1311 "Security Framework for Ubiquitous Sensor Networks". ITU-T, Geneva, February, 2011.

[27] www.zigbee.org.

[28] O. Younis, S. Fahmy. Distributed clustering in ad-hoc sensor networks: A hybrid, energy-efficient approach. Proceedings, IEEE INFOCOM, Hong Kong, China, 2004.

[29] S.Andreev, O.Galinina, Y.Koucheryavy. Energy-Efficient Client Relay Scheme for Machine-to-Machine Communication. IEEE Globecom 2011, Houston, TX, USA.

[30] A.Aziz, A.Salim, W.Osamy. Adaptive and Efficient Compressive Sensing based Technique for Routing in Wireless Sensor Networks. Proceedings, INTHITEN (IoT and its Enablers) conference. St.Petersburg, State University of Telecommunication, 3-4 June, 2013.

[31] T.Bhattasali, R.Chaki. A Survey of Recent Intrusion Detection Systems in Wireless Sensor Networks. Advanced in Network Security and Applications. Conference Proceedings of Fourth International Conference on Network security and Applications (CNSA 2011), Chennai, India, July 15-17, 2011.

[32] T.Bhattassali, R.Chaki, S.Sanyal. Sleep Deprivation Attack Detection in Wireless Sensor Networks. International Journal of Computer Applications, v.40, №15, February 2012.

[33] A.Koucheryavy, I.Bogdanov, A.Paramonov. The mobile Sensor Network Life-Time under Different Spurious Flows Intrusion. LNCS, Springer. 13 th NEW2AN, LNCS 8121, 28-30, August, 2013. 